\title{
NASAL CARRIAGE OF STAPHYLOCOCCUS AUREUS WITH SPECIAL EMPHASIS ON METHICILLIN- RESISTANT STAPHYLOCOCCUS AUREUS AMONG STUDENTS OF A SOUTH INDIAN MEDICAL COLLEGE - PREVALENCE AND ANTIBIOGRAM PATTERN
}

\author{
RADHAKRISHNA M*, AISHWARYA TANEJA, POOJA RAO \\ Department of Microbiology, Kasturba Medical College, Manipal University, Mangalore, Karnataka, India. \\ Email: manipuraradhakrishna@yahoo.com
}

Received: 04 June 2016, Revised and Accepted: 10 June 2016

\section{ABSTRACT}

Objective: There is paucity of information on carriage of Staphylococcus aureus and Methicillin Resistant S.aureus from developing nations including the medical students who could be the potential transmitters. Present study was aimed at establishing the prevalence and antibiogram pattern of S. aureus with special emphasis on MRSA among II year MBBS students of Kasturba Medical College, Mangalore.

Methods: A total of 148 students were participated in this study. Swabs taken from both anterior nares were processed, and the growth of S. aureus was confirmed by standard procedure. Further categorization of $S$. aureus into MRSA was done using cefoxitin disc diffusion method, along with the antibiogram to other common drugs.

Results: The number of strains of $S$. aureus isolated from our 148 participants was 78 with a percentage rate of 52.7 .0 . the 78 isolates of $S$. aureus, $9(11.5 \%)$ were MRSA. The overall MRSA carriage rate was $6.1 \%$.

Conclusion: The S. aureus and MRSA carriage rates recorded in this study were significantly higher when compared with other reported studies. It was observed that risk factors like use of antibiotics in past 6 months and snoring were found to be statistically significant in nasal carriage status of S. aureus. Out of nine MRSA carriers, six were found to be having the parents who were busy clinicians by occupation and the close contact with them could be the prime factor in the acquisition of MRSA carriage status.

Keywords: Staphylococcus aureus, MRSA, Nasal carriage, Medical students.

(C) 2016 The Authors. Published by Innovare Academic Sciences Pvt Ltd. This is an open access article under the CC BY license (http://creativecommons. org/licenses/by/4. 0/) DOI: http://dx.doi.org/10.22159/ajpcr.2016.v9s2.13274

\section{INTRODUCTION}

Staphylococcus aureus remains an important human pathogen responsible for health - care and community-associated infections, which also behaves as a commensal in healthy individuals, adopting the anterior nares as its ecological niche. Although primary $S$. aureus infections are not common, a great deal of the virulence from this organism occurs through cross-infection by spread from patient to patient in hospitals and other institutional settings. In contrast, healthy individuals have a small risk of contracting an invasive infection caused by $S$. aureus, but they can be carriers of the organism [1]. It is well known that nasal colonization constitutes a risk factor for subsequent severe infection but it also can be the source of transmission of this bacterium to other susceptible individuals [2]. Asymptomatic carriage of $S$. aureus in healthy individuals has been shown to have a high prevalence, especially in children, young adults, and health-care workers [3]. The incidence of community-acquired and hospital-acquired $S$. aureus infections has been rising with increasing emergence of drug-resistant strains called methicillinresistant S. aureus (MRSA) [4]. MRSA is an established pathogen in most health care facilities. Previously limited to hospitals, MRSA infections have been increasingly reported in the community $[5,6]$. A recent meta-analysis of 27 studies of the prevalence of communityacquired-MRSA among hospital patients that used clinical specimens, as opposed to surveillance cultures conducted at the time of admission, yielded a prevalence of MRSA of $30.2 \%$ [7]. A variety of studies have examined community prevalence of nasal carriage of S. aureus in diverse subpopulations such as adult outpatients, healthcare workers, college students, and injection drug users $[8,9]$. These are issues that may be very useful to clinicians when trying to decide the likelihood that a given patient has a staphylococcal infection and, if so, whether antibiotic coverage should be provided for resistant strains [5]. Exposure to microbes is an inherent risk of working in patient care settings. In view of its increasing incidence in the general population, acquisition of MRSA is a special concern for health-care workers [10]. Medical students represent an important portion of the healthcare personnel, and they are in frequent contact with patients. Thus, they are at risk of being colonized with different pathogens including $S$. aureus and of spreading them to susceptible patients. Different studies around the world have evaluated nasal carriage rates of $S$. aureus in medical students. Some of these studies reported that nasal carriage rates of $S$. aureus increased with greater exposure of students to the hospital environment [11-13]. No in detail study has analyzed whether this patient exposure creates a risk of MRSA carriage for Indian medical students. The aim and objective of the study was to evaluate the prevalence of nasal S. aureus, MRSA in particular, antibiotic susceptibility pattern and to correlate the risk factors for the carriage status among the $2^{\text {nd }}$ year medical students of our institution.

\section{METHODS}

The proposed study was conducted in the Microbiology Diagnostic Laboratory of Kasturba Medical College, Mangalore. This was a type of cross-sectional study. All consenting students of $2^{\text {nd }}$ year MBBS, the third term were randomly recruited into the study. The study proposal was submitted to the ethical review committee of the institution for approval. The age, sex, lifestyle, current skin infection/other illnesses and another relevant information about the participants was obtained 
in a pro forma designed for the said purpose. A total of 148 students were participated in this study. Swabs of both anterior nares of consenting persons were taken with a sterile swab stick moistened with sterile physiological saline. Processing of the samples was done immediately within $2 \mathrm{hrs}$ after collection. In the case of delay, more than $2 \mathrm{hrs}$ the swabs were stored at $4^{\circ} \mathrm{C}$ for maximum $24 \mathrm{hrs}$. The swabs were inoculated onto mannitol salt agar (MSA), and the inoculated MSA was incubated at $37^{\circ} \mathrm{C}$ for $18-24 \mathrm{hrs}$. The growth of the organism was identified as $S$. aureus using standard tests such as colony morphology, gram stain, catalase test, and coagulase test [13]. The isolated strains of $S$. aureus were screened for methicillin resistance by modified Kirby-Bauer method using cefoxitin $(30 \mu \mathrm{g})$ disc on MuellerHinton agar (MHA) [14]. The MHA on which cefoxitin was placed was incubated aerobically at $37^{\circ} \mathrm{C}$ for $18 \mathrm{hrs}$. Isolates with inhibition zone diameter $\leq 21 \mathrm{~mm}$ around cefoxitin disc were considered MRSA strains [15]. Screening for vancomycin susceptibility was also done by the modified Kirby-Bauer method using vancomycin disc $(30 \mu \mathrm{g})$ on MHA incubated at $37^{\circ} \mathrm{C}$ for $24 \mathrm{hrs}$. Further confirmation of vancomycin susceptibility was done by minimum inhibitory concentration (MIC) estimation using vancomycin E-test strips. Antibiotic sensitivity to all $S$. aureus isolates against other antibiotics such as amoxicillin/ clavulanic acid, cotrimoxazole, ciprofloxacin, ceftriaxone, erythromycin, gentamicin, linezolid, penicillin, and teicoplanin were determined by modified Kirby-Bauer method. All inoculum on MHA was 4-6 hrs growth of pure isolates in Muller Hinton broth, with density equivalent to 0.5 McFarland turbidity standard. S. aureus ATCC 25923, MRSA ATCC 29213, MSSA ATCC 33591 were used as control. All antibiotic discs and the vancomycin E test strips were procured from HiMedia Laboratories Pvt. Limited, India. Antibiotic sensitivity testing and result interpretation was done according to CLSI Guidelines [16].

\section{Statistical analysis}

Results were compelled and tabulated, and all data were subjected to the Statistical Package Social Sciences SPSS version 17.0. The results were presented in the form of tables and graphs. Associations of different factors were done using Chi-square test. $\mathrm{p}<0.05$ was considered as significant.

\section{RESULTS AND DISCUSSION}

A total of 148 students of II MBBS, age range 19-22 years were screened for MRSA. 63 (42.6\%) were males and 85 (57.4\%) females (Table 1). The types of organisms isolated from the anterior nares of the participants have been shown in Table 2. S. aureus was predominant $(52.7 \%)$ followed by coagulase-negative Staphylococcus (28.4\%). Out of 148,78 students were positive for nasal carriage of $S$. aureus giving a carriage rate of $52.7 \%$. Nine out of 78 isolates of $S$. aureus were MRSA giving a percentage of 11.5. Overall, MRSA nasal carriage rate was $6.1 \%$ in this study. Out of nine, six MRSA isolates were from females and the rest three were from males. The antibiotic susceptibility pattern of $S$. aureus has been shown in Fig. 1. Whereas anti-biogram pattern of MRSA isolates has been shown in Fig. 2. 92\% of the isolates of $S$. aureus were resistant to penicillin, whereas resistance to amoxiclav and erythromycin were $15.4 \%$ and $54 \%$, respectively. The nine isolates of MRSA showed sensitivity of $100 \%$ to vancomycin, linezolid, ceftriaxone, gentamicin, and teicoplanin, 33.3\% to ciprofloxacin and cotrimoxazole. All the nine isolates of MRSA were resistant to penicillin, erythromycin, and amoxiclav. Further MIC to vancomycin in all the nine isolates of MRSA was found to be $<2 \mu \mathrm{g} / \mathrm{ml}$ by E-test. The repeat samples obtained from the participants confirmed that they were MRSA and expressed the same pattern of antibiotic susceptibility. MSA found to be very useful for the quick identification of $S$. aureus.

\section{DISCUSSION}

Nasal carriage of $S$. aureus among II year MBBS students of Kasturba Medical College, Mangalore, according to the result of this study was $52.7 \%$. Out of 78 isolates of $S$. aureus, nine were MRSA positive with the percentage of 11.5. The overall MRSA carriage rate was $6.1 \%$. The $S$. aureus and MRSA carriage rates recorded in this study were significantly higher when compared with other reported studies. Different studies around the world have evaluated nasal carriage rates of $S$. aureus and MRSA in pre-clinical medical students. One of the studies from India by involving pre-clinical students of Malaysian base revealed nasal carriage rate of $23.7 \%$ of $S$. aureus and no MRSA [18]. Yet another study from Thailand disclosed $29.68 \%$ of S.aureus nasal carriage rate and again with zero percentage of MRSA [19]. Whereas the outcome of two more studies from pre-clinical students of Duhok University and Saudi Arabia were $9.2 \% ; 18.7 \%$ of S. aureus and $4.6 \%$; $6.7 \%$ of MRSA respectively $[20,21]$. It was observed that risk factors like use of antibiotics in past 6 months and snoring were found to be

Table 1: Gender wise distribution of participants, carriage status of $S$. aureus and MRSA

\begin{tabular}{llll}
\hline Gender & $\begin{array}{l}\text { Number of } \\
\text { participants (\%) }\end{array}$ & $\begin{array}{l}\text { S. aureus } \\
\text { carriers (\%) }\end{array}$ & $\begin{array}{l}\text { MRSA } \\
\text { carriers (\%) }\end{array}$ \\
\hline Male & $63(42.6)$ & $33(42.3)$ & $3(33.3)$ \\
Female & $85(57.4)$ & $45(57.7)$ & $6(66.6)$ \\
Total & 148 & $78(52.7)$ & $9(6.1)$ \\
\hline
\end{tabular}

MRSA: Methicillin-resistant Staphylococcus aureus,

$S$ aureus: Staphylococcus aureus

Table 2: Nature of organisms isolated from anterior nares

\begin{tabular}{lll}
\hline S. No. & Organisms isolated & Frequency (\%) \\
\hline 1 & S. aureus & $78(52.70)$ \\
2 & CONS & $42(28.3)$ \\
3 & Diphtheroids & $9(6.10)$ \\
4 & No growth & $19(12.83)$ \\
\hline S. aureus: Staphylococcus aureus, CONS: Coagulase negative Staphylococcus
\end{tabular}

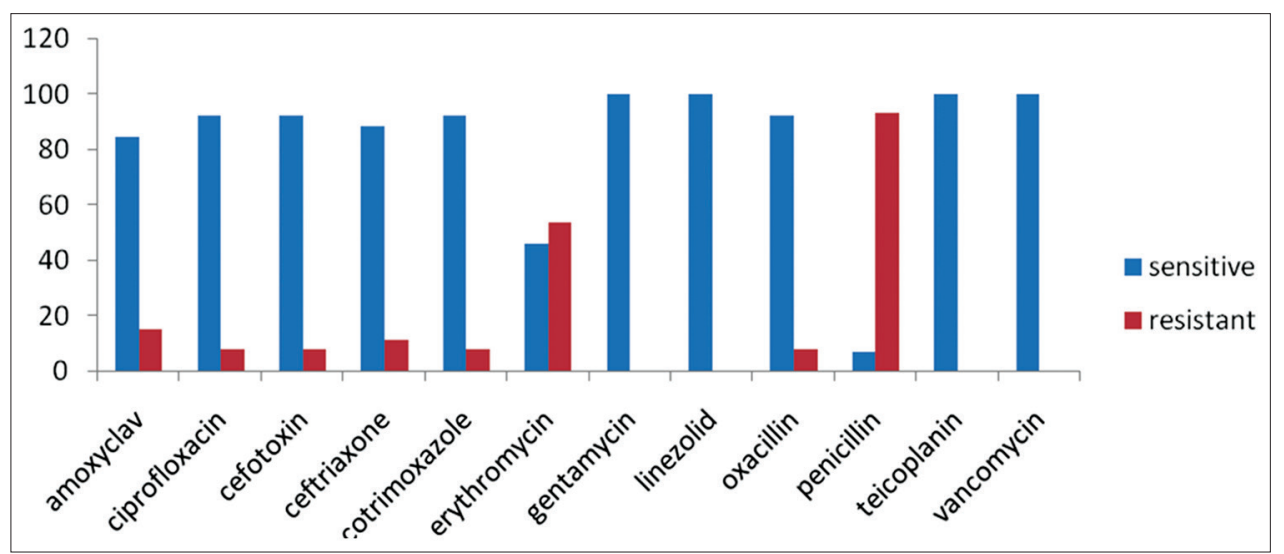

Fig. 1: Antibiotic susceptibility profile of Staphylococcus aureus isolates 


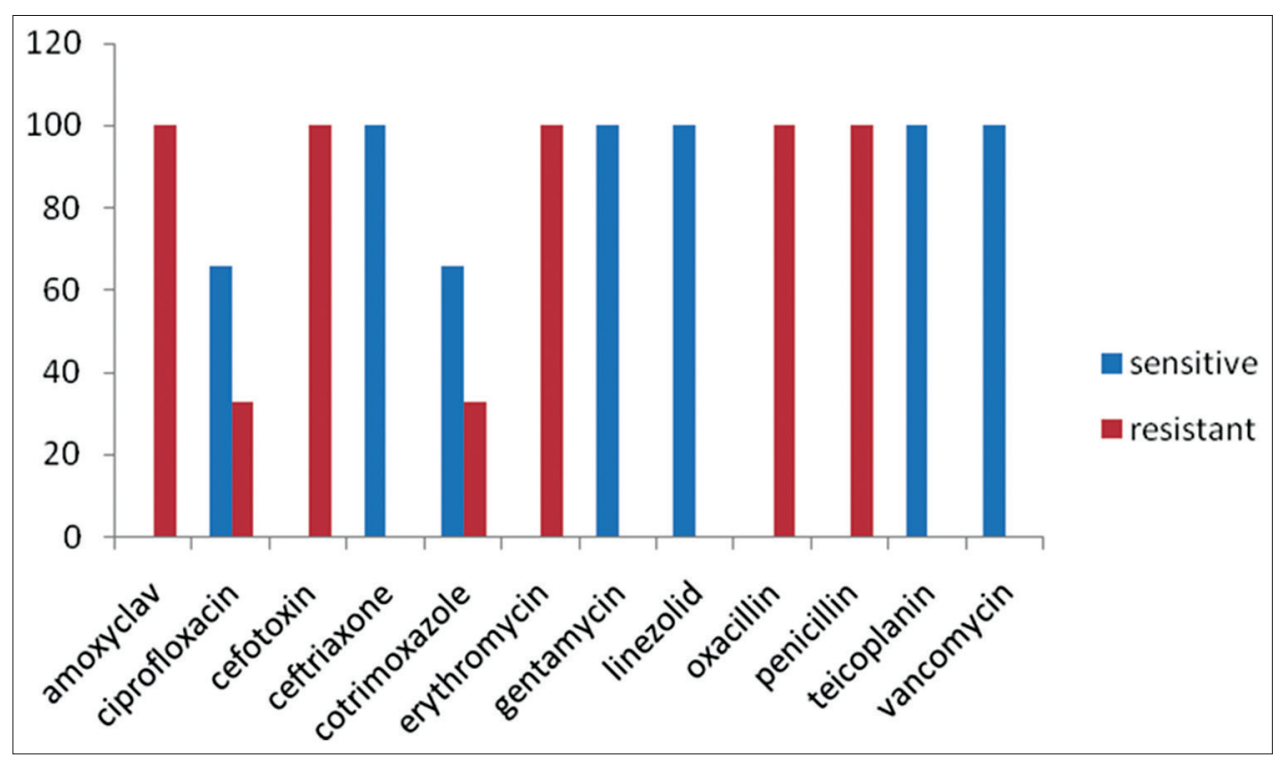

Fig. 2: Antibiotic susceptibility profile of methicillin-resistant Staphylococcus aureus isolates

statistically significant in nasal carriage status of $S$. aureus among the students $(\mathrm{p}<0.05) .56 \%$ of the student population who were the carriers of $S$. aureus had used antibiotics in the past 6 months and $42 \%$ of the $S$. aureus carriers had snoring problem. In this study with the identified nine MRSA carriers, there was no specific correlation with any risk factors for the acquisition of carriage status. Out of nine MRSA carriers, six were found to be having the parents who were busy clinicians by occupation. Therefore it is possible that the acquisition of MRSA carriage status could be due to the close contact with their parents who are in medical practice. It is necessary to screen the parents of those MRSA carriers to know whether those are also the carriers of MRSA or not. Another MRSA carrier was found to be in close contact with a student who was found to be MRSA carrier in this study. In remaining two MRSA carriers, the reason for the carriage status may be either community acquired or due to contact with the patients or carriers such as health-care workers in the hospital where they have been posted since last 1 year. Repeated isolation of MRSA with the same anti-biogram pattern in all the nine individuals confirmed that the MRSA carriage status was not transient. Therefore, it is desirable to treat those identified MRSA carriers among the student population so that they will not be the source of infection to the patients or other individuals. Since all the strains of MRSA were sensitive to vancomycin, teicoplanin, linezolid it will not be difficult to treat them. Aiming at $0 \%$ carriage rate these students may be treated with mupirocin and further screened for MRSA carriage status to check the efficacy of treatment.

There are published evidence pointing out that health-care workers more likely act as vectors and not as the main sources of MRSA transmission; thus emphasizing that good hand hygiene practices remain essential to control the spread of MRSA $[22,23]$. Eradication of MRSA colonization from patients and healthy carriers has been tried with topical and oral antibiotics. The international guidelines recommend the use of mupirocin for nasal decolonization in certain groups of patients and health-care workers colonized with MRSA [24]. The limitation is, this antibiotic is only effective at removing the bacterium from the nose over a few weeks, and nasal relapses are common within several months [25]. Furthermore, clinical studies have found little or no efficacy of mupirocin treatment in preventing nosocomial S. aureus infections [26]. Another shortcoming for this antibiotic is the increasing trend in resistance and the subsequent reduction in effectiveness [27]. It is recommended to educate and treat those MRSA carriers after taking their consent and repeat the nasal swab culture to check the efficacy of treatment in the eradication of
MRSA carriage status. However, periodic screening for MRSA should be an on-going practice in medical student population.

\section{ACKNOWLEDGMENT}

Authors would like to thank the Manipal University for Providing Facilities for carrying out this Research Work and the Technical Staff of Department of Microbiology, KMC, Mangalore. This work was supported by ICMR STS 2012 project.

\section{REFERENCES}

1. Kluytmans JA, Wertheim HF. Nasal carriage of Staphylococcus aureus and prevention of nosocomial infections. Infection 2005;33(1):3-8.

2. Peacock SJ, de Silva I, Lowy FD. What determines nasal carriage of Staphylococcus aureus? Trends Microbiol 2001;9(12):605-10.

3. Lin YC, Lauderdale TL, Lin HM, Chen PC, Cheng MF, Hsieh KS, et al. An outbreak of methicillin-resistant Staphylococcus aureus infection in patients of a pediatric intensive care unitand high carriage rate among health care workers. J Microbiol Immunol Infect 2007;40(4):325-34.

4. Deresinski S. Methicillin-resistant Staphylococcus aureus: An evolutionary, epidemiologic, and therapeutic odyssey. Clin Infect Dis 2005;40(4):562-73.

5. Chambers HF. The changing epidemiology of Staphylococcus aureus? Emerg Infect Dis 2001;7(2):178-82.

6. Naimi TS, LeDell KH, Como-Sabetti K, Borchardt SM, Boxrud DJ, Etienne J, et al. Comparison of community- and health care-associated methicillin-resistant Staphylococcus aureus infection. JAMA 2003;290(22):2976-84.

7. Salgado CD, Farr BM, Calfee DP. Community-acquired methicillinresistant Staphylococcus aureus: A meta-analysis of prevalence and risk factors. Clin Infect Dis 2003;36(2):131-9.

8. Bischoff WE, Wallis ML, Tucker KB, Reboussin BA, Sherertz RJ. Staphylococcus aureus nasal carriage in a student community: Prevalence, clonal relationships, and risk factors. Infect Control Hosp Epidemiol 2004;25(6):485-91.

9. Eveillard M, Martin Y, Hidri N, Boussougant Y, Joly-Guillou ML. Carriage of methicillin-resistant Staphylococcus aureus among hospital employees: Prevalence, duration, and transmission to households. Infect Control Hosp Epidemiol 2004;25(2):114-20.

10. Chambers HF. The changing epidemiology of Staphylococcus aureus? Emerg Infect Dis 2001;7(2):178-82.

11. Albrich WC, Harbarth S. Health-care workers: Source, vector, or victim of MRSA? Lancet Infect Dis 2008;8(5):289-301.

12. Güçlü E, Yavuz T, Tokmak A, Behçet M, Karali E, Oztürk O, et al. Nasal carriage of pathogenic bacteria in medical students: Effects of clinic exposure on prevalence and antibiotic susceptibility. Eur Arch Otorhinolaryngol 2007;264(1):85-8.

13. Adesida SA, Abioye OA, Bamiro BS, Brai BI, Smith SI, Amisu KO, 
et al. Associated risk factors and pulsed field gel electrophoresis of nasal isolates of Staphylococcus aureus from medical students in a tertiary hospital in Lagos, Nigeria. Braz J Infect Dis 2007;11(1):63-9.

14. Cheesbrough M. District Laboratory Practice in Tropical Countries - Part 2. Cambridge: Cambridge University Press; 2008. p. 62-70.

15. Bauer AW, Kirby WM, Sherris JC, Turck M. Antibiotic susceptibility testing by a standardized single disk method. Am J Clin Pathol 1966;45(4):493-6.

16. CLSI. Surveillance for Methicillin-resistant Staphylococcus aureus: Principles, Practices, and Challenges; A Report. CLSI Document X07-R. Wayne, PA: Clinical and Laboratory Standards Institute; 2010.

17. Clinical and Laboratory Standards Institute. NCCLS Performance Standards for Antimicrobial Disc Diffusion Tests; Approved Standards. CLSI Document M2-M9. $9^{\text {th }}$ ed. Wayne, PA: Clinical and Laboratory Standards Institute; 2006.

18. Santhosh DV, Shobha KL, Bairy I, Rao G, Anand KM, D'Souza J Nasal screening and survey of pre-clinical medical students from Malaysia for nasal carriage of coagulase positive MRSA and rate of nasal colonization with Staphylococcus species. J Clin Diagn Res 2007;1(6):494-9.

19. Treesirichod A, Hantagool S, Prommalikit O. Nasal carriage and antimicrobial susceptibility of Staphylococcus aureus among medical students at the HRH Princess Maha Chakri Sirindhorn Medical Center, Thailand: A cross sectional study. J Infect Public Health 2013;6(3):196-201.

20. Ma XX, Sun DD, Wang S, Wang ML, Li M, Shang H, et al. Nasal carriage of methicillin-resistant Staphylococcus aureus among preclinical medical students: Epidemiologic and molecular characteristics of methicillin-resistant S. aureus clones. Diagn Microbiol Infect Dis 2011;70(1):22-30.

21. Shadi AZ. Prevalence of methicillin-resistant Staphylococcus aureus nasal colonization among medical students in Jeddah, Saudi Arabia. Saudi Med J 2015;36(7):807-12.

22. Sax H, Allegranzi B, Uçkay I, Larson E, Boyce J, Pittet D. 'My five moments for hand hygiene': A user-centred design approach to understand, train, monitor and report hand hygiene. J Hosp Infect 2007;67(1):9-21.

23. WHO. WHO Guidelines on Hand Hygiene in Health Care (Advanced Draft): A Summary. Clean Hands are Safer Hands; 2005. Available from: http://www.who.int/patientsafety/events/05/HH en.pdf. [Last updated on 2005; Last cited on 2012 Apr 09].

24. Coia J, Duckworth G, Edwards DI, Farrington M, Fry C, Humphreys H, et al. Guidelines for the control and prevention of methicillin-resistant Staphylococcus aureus (MRSA) in healthcare facilities. J Hosp Infect 2006;63 Suppl 1:S1-44.

25. Doebbeling BN, Reagan DR, Pfaller MA, Houston AK, Hollis RJ, Wenzel RP. Long-term efficacy of intranasal mupirocin ointment A prospective cohort study of Staphylococcus aureus carriage. Arch Intern Med 1994;154(13):1505-8.

26. Kalmeijer MD, Coertjens H, van Nieuwland-Bollen PM, BogaersHofman D, de Baere GA, Stuurman A, et al. Surgical site infections in orthopedic surgery: The effect of mupirocin nasal ointment in a double-blind, randomized, placebo-controlled study. Clin Infect Dis 2002;35(4):353-8.

27. Simor AE, Stuart TL, Louie L, Watt C, Ofner-Agostini M, Gravel D, et al. Mupirocin-resistant, methicillin-resistant Staphylococcus aureus strains in Canadian hospitals. Antimicrob Agents Chemother 2007;51(11):3880-6. 